\title{
Corneal Diseases in Children in The Gambia
}

\author{
ROO Onabolu FWACS, FMC Oph, NA Iwuora FWACS, FMC Oph, W Ceesay MB.ChB D.O \\ Royal Victoria Teaching Hospital (RVTH), Banjul, The Gambia
}

\section{SUMMARY}

Background: Blindness surveys carried out in The Gambia in 1986 reported childhood blindness from non-trachomatous corneal diseases as the commonest cause of blindness in children. The major causes were, post measles keratopathy, vitamin A deficiency and ophthalmia neonatorum.

Aim: This study aims to find out what impact the Expanded Programme on Immunization and the development of human resources have had on the causes/pattern of corneal eye diseases in children in The Gambia.

Design: A prospective six-month hospital-based study.

Method: The eyes of all children aged 0-16 years presenting at the Royal Victoria Teaching Hospital (RVTH) in Banjul, The Gambia, from December 2005 to May 2006, were examined for aetiology of corneal eye diseases. All the children were treated according to the diagnosis.

Result: $A$ total of 169 children aged 0-16 years were seen during the study period. Seventy-one (42\%) had corneal diseases. Their ages ranged from 2 weeks to 16 years; mean 7.5 years and SD 3.1 years. The aetiology of corneal diseases were trauma in 23 (32.4\%) vernal kerato-conjunctivitis in 16 $(22.54 \%)$, congenital eye diseases in $12(16.9 \%)$, corneal infections in $12(16.9 \%)$, corneal scarring from unknown causes in $5(7.04 \%)$ and corneal dystrophy/degenerations in 3 patients. Ten patients (14\%) became bilaterally blind while 22 patients (31\%) suffered unilateral blindness, with congenital diseases and trauma being the causes respectively. Vernal kerato-conjunctivitis was a major cause of ocular morbidity.

Conclusion: Bilateral blindness is no longer due to preventable causes like measles, ophthalmia neonatorum and keratomalacia. The use of harmful traditional eye medication is a contributory factor to the severity of vernal keratoconjunctivitis.

Key words: corneal blindness, children, primary eye care

\section{INTRODUCTION}

The prevalence of blindness in children varies from $0.3 / 1000$ in wealthy countries to $1.2 / 1000$ in poor countries of the world. ${ }^{1}$ This disparity is due to corneal diseases from preventable causes like Vitamin A deficiency, measles and ophthalmia neonatorum. ${ }^{1,2}$ Poor socioeconomic development, non-availability of primary health care and eye care services in rural communities also contribute to the high prevalence of blindness. Reduction of childhood blindness is one of the priorities of Vision 2020. ${ }^{3}$ To achieve this objective, the elimination of corneal blindness from measles, ophthalmia neonatorum, Vitamin A deficiency has been targeted.

The 1986 survey of blindness in The Gambia recorded cataract blindness in adults as the most common cause of blindness followed by corneal diseases in children and adults. ${ }^{4}$ In order to address this issue, the government drew up a comprehensive eye care programme in collaboration with Sight Savers International. Eye care workers were trained to work in rural communities and there was a concurrent improvement in childcare. National immunization coverage of measles rose from $62 \%$ in 1986 to $93.8 \%$ in $1996 .^{5}$ A repeat survey of blindness ten years later in 1996 recorded a reduction in the prevalence of blindness from $0.70 \%$ to $0.4 \%{ }^{6}$ Age stratified prevalence in 1996 revealed $0.02 \%$ in the $0-9$ years age group, comparable to figures in economically advanced countries. This study aims to find the current aetiology of corneal diseases in children presenting at the Royal Victoria Teaching Hospital 20 years after the initial survey of blindness in the country.

\section{METHOD}

The study was approved by the RVTH board of ethics. The eyes of all children from 0-16 years presenting at RVTH from December 2005 to May 2006 were examined for aetiology of corneal eye diseases. The vision of older babies and preverbal children was roughly assessed using the preferential looking method. The visual acuity of verbal children, i.e. $>3$ years, were taken with the Snellen E-chart while the Snellen literate chart was used for literate children older than 5 years. The eyes of babies were examined under general anaesthesia if satisfactory diagnosis could not be obtained from bright pen torches and loupes. Older children were examined with slit lamps, and funduscopy was attempted in all the children. Corneal scraping in corneal ulcerations was done for five children who had not used any medication. These children, with others suspected of having bacterial ulcers that were inadequately treated, were given

*Correspondence: Dr OO Onabolu, Consultant Ophthalmologist, Ophthalmology Department, OACHS, PMB 2027, Sagamu, Ogun State, Nigeria • E-mail: tonionabolu@yahoo.com 
subconjunctival gentamicin 10mg (dose based on their ages) with $0.2 \mathrm{ml}$ plain xylocaine every 48 hrs for 5 doses. Guttae ciprofloxacin or gentamicin hourly, and mydriacyl 3 times daily were added. The medication was commenced while waiting for laboratory results. Those with dendritic staining ulcers had acyclovir eye ointment 5 times daily until the ulcers healed. All the children with Moorens ulcer were treated for hookworm infestation and peritomy was done in the affected eye. They also had topical hydrocortisone and chloramphenicol. Children with trauma had the cornea repaired, while those with keratoconus had conjunctiva flaps raised to cover the descematocoele. Children with congenital conditions were treated symptomatically. Those with glaucoma whose intraocular pressures could not be controlled with medication had trabeculectomy. Patients with acute conditions were followed up until the corneae healed, while those with chronic diseases were come back on regular visits.

\section{RESULTS}

A total of 169 children aged 0-16 years were seen during the study period. Seventy-one (42\%) had corneal diseases (table 1). There were 48 male and 23 female participants; with a male to female ratio of 2:1.04. Their ages ranged from 2 weeks to 16 years; mean 7.5 years SD 3.1. The aetiology of the corneal diseases were trauma in $23(32.4 \%)$, vernal kerato-conjunctivitis in $16(22.54 \%)$, congenital eye diseases in $12(16.9 \%)$, corneal infections in $12(16.9 \%)$, corneal scarring from unknown causes in 5 (7.04\%), while $3(4.22 \%)$ had corneal degeneration/dystrophy (table 2). In the congenital diseases, 7 babies had corneal opacities from late presentation of bulphthalmos, 1 had anterior segment dysgenesis, 2 micro-ophthalmos, 1 megalocornea and 1 corneal dermoid. There was a history of consanguinity in the parents of six children. Children with vernal conjunctivitis had mostly limbal and mixed types with moderate to severe intensity. Three children had huge gelatinous limbal papillae extending towards the centre of the cornea. This was associated with brush-shaped epithelial scarring of the cornea. Vernal kerato-conjunctivitis was found to be an important cause of ocular morbidity.
Table 1. Anatomical site and pathology of ocular diseases in children in RVTH, The Gambia

\begin{tabular}{lcc}
\hline Diseases & No. & $\%$ \\
\hline Cornea & 71 & 42 \\
Lens (cataract and & 31 & 18.3 \\
pseudophakia) & 26 & \\
Lids and conjunctiva & 15 & 15.4 \\
Uvea and hyphaema & 10 & 8.9 \\
Retina and optic nerve & 8 & 5.9 \\
Squints and refractive error & 6 & 4.7 \\
Globe and orbit & 2 & 3.6 \\
Secondary glaucoma & 169 & 1.2 \\
\hline Total & & 100.0 \\
\hline
\end{tabular}

Children with corneal ulcerations were treated for bacterial (8), viral (2) and Moorens ulcers (2). Identification of the bacteria was difficult because of prior use of antibiotics from stations of referral and difficulty in taking proper scraping from children. There was also the difficulty of identifying the bacteria in the laboratory and so only one child had a positive gram stain result. One child with pustules on the scalp was suspected of having staphylococcal corneal ulcers. No child was seen with postmeasles keratopathy nor was any identified with signs of Vitamin A deficiency. Two of the children with corneal scars were suspected to have had post-measles keratopathy and Vitamin A deficiency in the past. The children with bilateral keratoconus had severe visual impairment after the corneae perforated. Conjunctival flaps appeared to be a temporary measure to prevent perforation, however, despite this intervention, the two eyes of one child were perforated. Trauma was more common in male children at a ratio of 2.28:1. Injury was often sustained while throwing sticks to harvest fruits and at play on the football field. Overall, 10 children (14.08\%) became bilaterally blind while 22 (30.98\%) were unilaterally blind. Congenital diseases were the major causes of bilateral blindness while trauma was the major cause of unilateral blindness (table 3 ).

Table 2. Aetiology of corneal diseases in 71 children in The Gambia

\begin{tabular}{|c|c|c|c|c|c|c|}
\hline Aetiology & Male & Female & Total & $\%$ & Age Range & Mean \\
\hline Trauma & 16 & 7 & 23 & 32.4 & 3-13 years & 7.7 years, SD 3.22 \\
\hline Congenital & 8 & 4 & 12 & 16.9 & 2-108 months & 8.2 months, SD 30 \\
\hline Vernal conjunctivitis & 10 & 6 & 16 & 22.52 & $5-16$ years & 11.19 years, SD 3.6 \\
\hline Inflammatory / infective & 9 & 3 & 12 & 16.9 & $1-16$ years & 9.2 years, SD 5.04 \\
\hline Degeneration/dystrophy & 1 & 2 & 3 & 4.22 & $10-12$ years & 11.33 years, SD 1,15 \\
\hline Corneal scar & 4 & 1 & 5 & 7.04 & $8 \mathrm{mths}$ & 3.33 years, SD 3.1 \\
\hline Total & 48 & 23 & 71 & 100 & & \\
\hline
\end{tabular}


Table 3. Visual outcome in 101 eyes of 71 children with corneal diseases

\begin{tabular}{lcccccc}
\hline & Trauma & Vernal & $\begin{array}{c}\text { Con- } \\
\text { genital }\end{array}$ & $\begin{array}{c}\text { Inflam- } \\
\text { matory/ } \\
\text { Infection }\end{array}$ & $\begin{array}{c}\text { Dysgen- } \\
\text { eration/ } \\
\text { Dys- } \\
\text { trophy }\end{array}$ \\
\hline Inaccessible & 3 & 2 & 17 & 17 & 0 & 4 \\
$3 / 60,<3 / 60$ & 15 & 0 & 2 & 2 & 2 & 0 \\
$6 / 24-6 / 60$ & 4 & 12 & 2 & 2 & 2 & 1 \\
$6 / 6-6 / 18$ & 1 & 18 & 0 & 0 & 2 & 1 \\
\hline Total eyes & 23 & 32 & 21 & 13 & 6 & 6 \\
\hline
\end{tabular}

\section{DISCUSSION}

Corneal scarring from inflammation and ulceration was an important cause of blindness in children before the advent of immunization and good antenatal care. ${ }^{7}$ Studies from schools for the blind in the poorer countries of the world also document these findings, ${ }^{8}$ however, improvement in antenatal care and child survival programmes in these countries are gradually been reflected in ophthalmology clinics.9 Our study seems to confirm that measles keratopathy and ophthalmia neonatorum as causes of blindness have decreased. Two of the older children with corneal scars were suspected of having had measles in the past while one child was suspected of having suffered from Vitamin A deficiency. Although babies were treated for ophthalmia neonatorum, prompt presentation and aggressive management prevented any sequelae. In The Gambia, the presence of eye care workers in rural areas as community ophthalmic nurses enables mothers to access treatment without travelling far.

Children with microbial corneal ulcers presented late to the teaching hospital. Some of them had had prior treatment elsewhere but discontinued. Difficulty in identifying the causative organism resulted in prolonged morbidity. In the study of corneal ulcers by Leck et al., 31\% in India and 50\% in Ghana had no laboratory diagnosis and were treated based on the clinical signs. ${ }^{10}$ Clinical signs like pustules on the head for staphylococcal ulcers and dendritic staining were clues to the causes in three children in this study. The other children were treated aggressively with antibiotics. There is a need to improve on laboratory diagnosis of corneal disease in developing countries.

Moorens ulcer in this age group tended to be bilateral and exhibited a typical aggressive nature. Despite treatment for hookworm, topical steroid antibiotic combination and peritomy, they still perforated later. Moorens ulcer is believed to have an autoimmune disposition. There is a deficiency of suppressor T cells, increased level of IgA, tissue fixed immunoglobulins and compliment in the conjunctiva epithelium and peripheral cornea. ${ }^{11}$ Immunosuppressives such as cyclophosphamide, methotrexate and cyclosporine were not available for use. Generally, treatment of bilateral Moorens ulcer has not been satisfactory as reported in the literature. ${ }^{12}$ Vision was lost in both eyes of one patient.

Trauma was the commonest cause of corneal disease and the most common cause of blindness in those whose vision could be accessed. Previous work on ocular trauma has shown that it is commoner in children and young adults and that males are more predisposed to trauma. ${ }^{13}$ In this study, $16(69.56 \%)$ of the 23 children with ocular trauma were male and at least $15(65.21 \%)$ became blind in the affected eye. Dandona et al., in their study of corneal blindness in Southern India, found trauma and keratitis in childhood to be an important cause of uniocular blindness. ${ }^{14}$ In this study, children got injured from throwing sticks to harvest fruits and from scuffles. To reduce the incidence of this type of injury, this issue is being seriously addressed in radio broadcasts during the mango season. Strict parental supervision will go a long way in decreasing ocular injuries in children.

Congenital malformations of the cornea were found in $16.9 \%$ of the children in this study. It is estimated that congenital malformations of the eye are found in $2-3 \%$ of all live births. ${ }^{15}$ In a ten-year study of congenital eye diseases in Abuja (Nigeria) Babalola and Babalola ${ }^{16}$ found that $30.5 \%$ of all children less than 1 year that were seen had congenital anomalies and $7.3 \%$ had ophthalmia neonatorum. The low incidence of preventable causes of ocular diseases in this age group was probably due to the high socioeconomic status of the city and the fact that the facility was a private hospital. As access to health facilities improve associated with an improvement in socioeconomic status, causes of preventable corneal blindness will decrease. It is expected that this will be the trend in The Gambia in the near future.

In this study, anterior chamber dysgenesis and congenital glaucoma were the predominant causes of congenital corneal disease as opposed to Babalola's study where nasolacrimal duct obstruction was the commonest. Patients with congenital glaucoma had dense corneal opacities that rarely cleared after surgery. There is a problem of bringing the babies to tertiary centres early. Perhaps the disease manifested early in life thereby worsening the prognosis. The parents were young and one could not find any teratogen responsible. What was evident was a high consanguinity in the community. The malformations often affected the two eyes with the children becoming blind.

Vernal kerato-conjunctivitis was an important cause of ocular morbidity and severe visual impairment in children in The Gambia. In previous studies, vernal and allergic kerato-conjunctivitis were the commonest ocular disease in eye outpatient clinics in sub-Saharan Africa, affecting mainly 
the paediatric population. ${ }^{16,17}$ History of atopy was rare. The limbal type affected the cornea severely such that the central corneae were infiltrated with giant gelatinous papillae and scars, in agreement with previous findings. ${ }^{17}$ Some patients had bathed the eyes with local herbs complicating the clinical picture. These herbs might have been deleterious to the cornea. ${ }^{18}$ The chronicity of the disease would be a motivating factor to consult traditional healers. Children with such severe disease often rely on topical steroids for relief of their symptoms. There is an ongoing research into the use of amniotic membrane grafts for these recalcitrant cases. $^{19}$

The two children with keratoconus did not have the opportunity of corneal graft which had a deleterious effect on the eyes. Laser management with intacs implantation was not available either. ${ }^{20}$ Corneal grafting should be included as part of ophthalmic surgical procedures in tertiary centres. ${ }^{3}$

In conclusion, the major cause of bilateral corneal blindness in children in The Gambia is congenital ocular malformation. This study has shown that good primary health care as well as eye care can reduce and eradicate preventable corneal blindness in children.

\section{REFERENCES}

1. Gilbert C. New issues in childhood blindness. Community Eye Health Journal 2001; 14: 53-56.

2. Sandford-Smith JH. Corneal ulceration following measles in Nigerian children. Br J Ophthalmol 1979; 63: 720-729.

3. Gilbert C, Forster A. Childhood blindness in context of vision 2020-the right to sight. Bull World Health Organ 2001; 79: 227 32.

4. Faal H, Minassian D, Sowa S, Forster A. National Survey of blindness and low vision in The Gambia. Br J Ohthalmol1989; 73: 82-87.

5. Immunization Coverage 1976-2003. Ministry of Health, The Gambia.
6. Survey of Blindness in The Gambia. 1996. Royal Victoria Teaching Hospital, Banjul.

7. Onabolu OO. Post measles keratopathy-A recurring problem in Nigerian children. Nig J Paed 1999; 26: 41-43.

8. Ezegwu IR, Umeh RE, Ezepue UF. Causes of childhood blindness: Results from schools for the blind in eastern Nigeria. Br J Ophthalmol 2003; 87: 20-23.

9. Bodunde OT, Onabolu OO. Pattern of ophthalmic admissions into children's ward OSUTH Sagamu. Nig J Paed 2002; 10: 3234.

10. Leck AK, Thomas PA, Hagan M, Kahamuthy J, Ackuaku E, John $\mathrm{M}$ et al. Aetiology of suppurative corneal ulcers in Ghana and south India and epidemiology of fungal keratitis. Br J Ophthalmol 2002; 86: 1211-1215.

11. BCS Course. Sec 8, chapter 9.Clinical approach to immune related disorders of the external eye. 2008-2009: 1211-1215.

12. Sangwan VS, Zafirakis P, Foster CS. Moorens ulcer current concepts in management. Indian J Ophthalmol 1997; 45: 7-17.

13. Olurin O. Eye Injuries in Nigeria. Am J Ophthalmol 1971; 72: 159-166.

14. Dandona R, Dandona L. Corneal blindness in a southern Indian population: Need for health promotion strategies. $\mathrm{Br}$ J Ophthalmol 2003; 87: 133-141.

15. BCS Series Section 6, Chapter 15. Congenital anomalies 20082009: 195.

16. Babalola OE, Babalola BI. Congenital eye diseases in Abuja, Nigeria. Nigerian Journal of Ophthalmology 2004; 12: 45-49.

17. Medi Kawuna. The clinical picture of vernal keratopathy in Uganda. Community Eye Health Journal 2001; 40: 66-67.

18. Dekkers N.W.H.M. The cornea in measles. Dr W Junk Pub, The Hague, 1981.

19. Sangwan VS. LV Prasad Eye Institute, Hyderabad India. Personal communication 2006.

20. Ertan A, Kamburoglu G. Intacs implantation using a femtosecond laser for management of keratoconus; Comparison of 306 cases in different stages. J Cat Refr Surg 2008; 34: 1521-1526. 